Pacific Journal of Mathematics

ON A THEOREM OF S. BERNSTEIN 


\section{ON A THEOREM OF S. BERNSTEIN}

\section{A. Granas, R. B. Guenther and J. W. Lee}

In 1912, S. Bernstein, in the first part of his memoir [2] devoted to the boundary value problems arising in calculus of variations, established sufficient conditions for the unique solvability of the Dirichlet problem for the equation $y^{\prime \prime}=$ $f\left(t, y, y^{\prime}\right)$. Our aim is to present a result which extends the scope of the Bernstein theorem and to show that the generalization obtained can be carried over (with only minor adjustments in the proof) to the case of all important boundary value problems which arise in applications.

1. Introduction. In this paper we study the existence and uniqueness problems for a second order differential equation of the form

$$
\left(^{*}\right) \quad y^{\prime \prime}=f\left(t, y, y^{\prime}\right), \quad 0 \leqq t \leqq 1 \text {, }
$$

subject to certain boundary conditions, labeled (I)-(VII) below. These boundary conditions include the Dirichlet, Neumann, and periodic ones as well as the so-called elastic or Sturm-Liouville boundary conditions. We only treat in detail homogeneous boundary conditions; however, the case of inhomogeneous boundary conditions can be treated similarly to the homogeneous case. See $7(d)$.

The Dirichlet, Neumann, and periodic boundary conditions are, respectively,

$$
\begin{aligned}
\text { (I ) } y(0) & =0, & y(1) & =0 ; \\
\text { (II) } y^{\prime}(0) & =0, & y^{\prime}(1) & =0 ; \\
\text { (III) } y(0) & =y(1), & y^{\prime}(0) & =y^{\prime}(1) .
\end{aligned}
$$

The problem of solving the differential equation $\left({ }^{*}\right)$ subject to the boundary conditions (I) will be referred to as problem (I). Similar notation is used for the other problems.

We always assume that $f(t, y, p)$ is defined and continuous on $[0,1] \times R \times R$. By a solution to problem (I), we mean a function $y \in C^{2}[0,1]$ which satisfies the differential equation and boundary conditions. Likewise, we seek $C^{2}[0,1]$ solutions to the other problems.

In 1912, S. Bernstein established the following theorem in [2] for problem (I):

Assume $f=f(t, y, p)$ is continuous, has continuous partial derivatives $f_{y}$ and $f_{p}$, and satisfies

(i) $f_{y} \geqq k>0$

(ii) $|f(t, y, p)| \leqq A(t, y) p^{2}+B(t, y)$, 
where $A, B$ are functions bounded on each compact subset of $[0,1] \times R$ and $k$ is a constant. Then there exists exactly one solution $y \in C^{2}[0,1]$ to problem (I).

The purpose of this note is to present a generalization of the Bernstein theorem (which permits the treatment of equations such as $y^{\prime \prime}=y^{3}+y^{\prime 2}+1$ to which the original result does not apply) and to show that Bernstein's result can be extended to many other important boundary value problems. For purposes of comparison, we state the following extension of Bernstein's theorem (see Theorems (4.1), (5.2), and (5.3)).

Assume $f=f(t, y, p)$ is continuous and satisfies:

(i) There is a constant $M \geqq 0$ such that $y f(t, y, 0)>0$ for $|y|>M$;

(ii) $|f(t, y, p)| \leqq A(t, y) p^{2}+B(t, y)$ with $A, B$ as above. Then:

(a) there exists a solution $y \in C^{2}[0,1]$ to problem (I);

(b) there exists a solution $y \in C^{2}[0,1]$ to problems (II) and (III);

(c) moreover, if the partial derivatives $f_{y}$ and $f_{p}$ exist, are bounded, and $f_{y} \geqq 0$, then the solution in (a) is unique and any two solutions in (b) differ by a constant.

In $\S 5$ uniqueness theorems for problems (I)-(III) are established under a monotonicity requirement on $f$ rather than the differentiability assumptions in (c) above. Also, existence and uniqueness results are established for boundary value problems with SturmLiouville boundary conditions in $\S 6$.

Our discussion of existence and uniqueness questions proceeds as follows: The existence discussion falls into three parts. First sufficient conditions on $f$ are given which imply an a priori bound on a solution $y$ to one of the above problems. Next, assuming an a priori bound on $y$, an a priori bound on $y^{\prime}$ is obtained. Finally, the existence of a solution is obtained by applying a topological technique. Uniqueness is treated as a separate issue.

REMARK. For problem (I) we are seeking solutions $y \in C^{2}[0,1]$. It is more natural to seek solutions $y \in C^{2}(0,1) \cap C[0,1]$. However, the a priori bounds derived below, together with the assumptions on $f(t, y, p)$ imply, a priori, that $y$ must be in $C^{2}[0,1]$. Thus no generality is gained by considering solutions in the class $C^{2}(0,1) \cap$ $C[0,1]$. This comment also applies to the other problems. A similar remark is also pertinent in the uniqueness discussion.

2. A priori bounds on solutions. In this section a priori bounds are established for solutions of problems (I), (II), and (III). 
Let $y$ be a solution to $(*)$ and $r(t)=[y(t)]^{2}$. If $r$ has a positive maximum at $t_{0}$ in $[0,1]$ where, $y\left(t_{0}\right)>0$ say, then

$$
0 \geqq r^{\prime \prime}\left(t_{0}\right)=2 y\left(t_{0}\right) f\left(t_{0}, y\left(t_{0}\right), 0\right) \text {. }
$$

This motivates (2.1).

Lemma 2.1. Suppose there is a constant $M \geqq 0$ such that

$$
y f(t, y, 0)>0 \text { for }|y|>M .
$$

If $y$ is a solution to the differential equation $\left(^{*}\right)$ and $|y|$ does not achieve its maximum value at $t=0$ or 1 , then

$$
|y(t)| \leqq M \quad \text { for } t \text { in }[0,1]
$$

Proof. Under the conditions of the lemma $|y|$ must achieve a positive maximum at a point $t_{0}$ in $(0,1)$. Suppose $y\left(t_{0}\right)>0$. Then

$$
\begin{aligned}
& 0 \geqq y^{\prime \prime}\left(t_{0}\right)=f\left(t_{0}, y^{\prime}\left(t_{0}\right), 0\right), \\
& 0 \geqq y\left(t_{0}\right)=f\left(t_{0}, y^{\prime}\left(t_{0}\right), 0\right),
\end{aligned}
$$

and the last inequality also holds if $y\left(t_{0}\right)<0$. By our basic assumption on $f$ it follows that $\left|y\left(t_{0}\right)\right| \leqq M$. The lemma is proved.

LEMMA 2.2. If $f$ and $M$ satisfy the hypothesis of Lemma 1, then any solution $y$ to problem (I), (II) or (III) satisfies

$$
|y(t)| \leqq M, \quad t \text { in }[0,1] \text {. }
$$

Proof. For the Dirichlet problem (I) the assertion follows directly from (2.1).

Let $y$ be a solution to either problem (II) or (III). We claim:

(i) If $|y|$ assumes its maximum for $t_{0}=0$ or $t_{0}=1$, then $\left|y\left(t_{0}\right)\right| \leqq M$.

Clearly, (2.1) and (i) imply that $|y(t)| \leqq M$ for $t$ in $[0,1]$. It remains to prove (i) for problems (II) and (III).

Let $y$ be a solution to problem (II) for which $y(0)$ is the maximum value of $y$. We show that $y(0)>M$ is impossible. Indeed, if $y(0)>M$, then

$$
y(0) y^{\prime \prime}(0)=y(0) f(0, y(0), 0)>0 .
$$

If $y(0)>0$ then $y^{\prime \prime}(0)>0$ and so $y^{\prime}(t)$ is strictly increasing near $t=0$. Then $y^{\prime}(t)>y^{\prime}(0)=0$ for $t>0$ near zero, $y$ is strictly increasing near 0 , and $y(0)=|y(0)|$ is not the maximum of $|y|$ on $[0,1]$, a contradiction. Likewise, if $y(0)<0$ a similar contradiction arises. Thus, $|y(0)| \leqq M$. The corresponding assertion for $y(1)$ is proved 
similarly. This proves (i) for the Neumann problem.

Consider the periodic problem (III). If $y^{\prime}(0)=y^{\prime}(1) \neq 0$ it follows from $y(0)=y(1)$ that $|y|$ cannot achieve its maximum at $t_{0}=0$ or 1 . Since by (i), $|y|$ is assumed to achieve its maximum for $t_{0}=0$ or $t_{0}=1$, we must have $y^{\prime}(0)=0$. Then $y$ satisfies problem (II) and, hence, $|y(0)|=|y(1)| \leqq M$. This completes the proof.

The following corollaries are special cases of (2.2). They give conditions on $f$ which are easily checked in practice and imply that $y f(t, y, 0)>0$ for $|y|>M$.

CoRollary 2.3. Suppose $n$ is an odd integer,

$$
\frac{\partial^{k} f}{\partial u^{k}}(t, u, 0), \quad k=0, \cdots, n
$$

exist and are continuous, and that there is a constant $M \geqq 0$ such that

$$
\frac{\partial^{n} f}{\partial u^{n}}(t, u, 0) \geqq k \quad \text { for } \quad|u|>M, \quad k>0 \quad a \text { constant. }
$$

Then any solution $y$ to one of problems (I), (II) or (III) satisfies

$$
|y(t)| \leqq M \quad \text { for } t \text { in }[0,1]
$$

REMARK. These conditions are satisfied in particular when

$$
f(t, u, p)=\sum_{k=0}^{n} a_{k}(t, p) u^{k},
$$

$n$ is odd, and $a_{n}(t, 0)>0$ for $t$ in $[0,1]$, and $a_{l k}(t, p)$ is continuous for $k=0,1, \cdots, n$.

CoROLlaRY 2.4. Suppose $f(t, u, p)=u \alpha(t, u, p)+\beta(t, u, p)$ and that there is a constant $M \geqq 0$ such that for $|u|>M$,

$$
\alpha(t, u, 0)>0 \text { and } \frac{\beta(t, u, 0)}{u \alpha(t, u, 0)}<1 .
$$

Then any solution to problems (I), (II) or (III) satisfies

$$
|y(t)| \leqq M \quad \text { for } t \text { in }[0,1]
$$

3. A priori bounds for derivatives. In this section a priori bounds are established for the derivatives of solutions.

Lemma 3.1. (i) Suppose there is a constant $M>0$ such that 
$|y(t)| \leqq M$ for $0 \leqq t \leqq 1$ and for each solution $y \in C^{2}[0,1]$ to the differential equation $\left({ }^{*}\right)$.

(ii) Suppose there exist constants $A, B>0$ such that

$$
|f(t, u, p)| \leqq A p^{2}+B
$$

for all $(t, u)$ in $[0,1] \times[-M, M]$. Then there is a constant $\tilde{M}$ depending only on $M, A, B$ such that

$$
\left|y^{\prime}(t)\right| \leqq \tilde{M}, \quad t \text { in }[0,1],
$$

for each solution $y$ of $\left(^{*}\right)$ whose derivative vanishes at least once in $[0,1]$.

Proof. Since $y^{\prime}$ vanishes at least once in $[0,1]$, each point $t$ in $[0,1]$ for which $y^{\prime}(t) \neq 0$ belongs to an interval $[\mu, \nu]$ such that $y^{\prime}$ maintains a fixed sign on $[\mu, \nu]$ and $y^{\prime}(\mu)$ and/or $y^{\prime}(\nu)$ is zero. To be definite, assume that $y^{\prime}(\mu)=0$ and $y^{\prime} \geqq 0$ on $[\mu, \nu]$. Then from (ii),

$$
\frac{2 A y^{\prime} y^{\prime \prime}}{A y^{\prime 2}+B} \leqq 2 A y^{\prime}
$$

and integrating from $\mu$ to $t$ yields

$$
\begin{aligned}
\log \frac{A y^{\prime 2}(t)+B}{B} \leqq 4 A M & \\
& \left|y^{\prime}(t)\right| \leqq\left\{\frac{B}{A}\left(e^{4 . A M}-1\right)\right\}^{1 / 2} \equiv \widetilde{M},
\end{aligned}
$$

where $\widetilde{M}$ is defined by the last equality. The other possibilities that might occur are treated similarly and the same bound on $\left|y^{\prime}(t)\right|$ is obtained.

The boundary conditions for problems (I)-(III) imply that the derivative of each solution to one of these problems must vanish at least once in $[0,1]$. Thus, (2.2) and (3.1) imply:

Proposition 3.2. Suppose there is a constant $M \geqq 0$ such that $u f(t, u, 0)>0$ for $|u|>M$ and that there are functions $A(t, u)$, $B(t, u)>0$, bounded on compact subsets in $[0,1] \times R$, such that

$$
|f(t, u, p)| \leqq A(t, u) p^{2}+B(t, u) \text {. }
$$

Then there is a constant $\tilde{M}>0$ such that for any solution to problems (I), (II) or (III)

$$
|y(t)| \leqq M, \quad\left|y^{\prime}(t)\right| \leqq \widetilde{M} \quad \text { for } t \text { in }[0,1] .
$$


We will treat other types of growth conditions in a subsequent paper. Here we simply indicate one result in this direction.

Proposition 3.3. (i) Suppose there is a constant $M \geqq 0$ such that for any solution y to problem (II),

$$
|y(t)| \leqq M \quad \text { for } t \text { in } \quad[0,1]
$$

(ii) Suppose there is a constant $M_{1}$ such that

$$
|f(t, u, p)|>0 \quad \text { for } \quad|p|>M_{1} .
$$

Then there is a constant $\tilde{M}$ such that

$$
\left|y^{\prime}(t)\right| \leqq \tilde{M} \quad \text { for } t \text { in }[0,1] .
$$

Next suppose (i) holds for solutions to problem (III), $f$ satisfies (ii) and

(iii) $f(0, u, p)=f(1, u, p)$ for all $(u, p)$. Then there is a constant $\widetilde{M}$ such that

$$
\left|y^{\prime}(t)\right| \leqq \tilde{M} \quad \text { for } t \text { in }[0,1],
$$

for each solution $y$ to poblem (III).

Proof. Let $y$ be a solution to the Neumann problem. If $y^{\prime}$ has a nonzero maximum or minimum value at $t_{0}$, then $t_{0}$ is in $(0,1)$ and

$$
0=y^{\prime \prime}\left(t_{0}\right)=f\left(t_{0}, y\left(t_{0}\right), y^{\prime}\left(t_{0}\right)\right)
$$

which implies $\left|y^{\prime}\left(t_{0}\right)\right| \leqq M_{1}=\tilde{M}$. This establishes the result for problem (II).

Let $y$ be a solution to the periodic problem (III). In view of the boundary conditions, the form of the differential equation $\left(^{*}\right)$, and the periodicity of $f$ in $t$, each solution $y \in C^{2}[0,1]$ can be extended to a 1-periodic, $C^{2}$-solution to $\left(^{*}\right)$ on $(-\infty, \infty)$. Now the proof can be completed as for the Neumann problem.

REMARK. Suppose $f(t, u, p)=u \alpha(t, u, p)+\beta(t, u, p)$ satisfies the hypothesis of (2.4). Then (i) of (3.3) holds, and (ii) will hold if

$$
\frac{\alpha(t, u, p)}{\beta(t, u, p)} \longrightarrow 0 \quad \text { as } \quad|p| \longrightarrow \infty
$$

uniformly for $(t, u)$ in $[0,1] \times[-M, M]$.

The existence proof in $\S 4$ requires a priori bounds for the following family of problems 


$$
\left(\mathscr{H} \mathcal{C}_{\lambda}\right)\left\{\begin{array}{l}
y^{\prime \prime}-y=\lambda\left[f\left(t, y, y^{\prime}\right)-y\right], \\
y \in \mathscr{B},
\end{array}\right.
$$

depending on the parameter $\lambda, 0 \leqq \lambda \leqq 1$. Here $\mathscr{B}$ denotes the class of functions in $C^{2}[0,1]$ satisfying either boundary conditions (I), (II), or (III). Notice that the family $\mathscr{H}_{2}$ joins either problem (I), (II), or (III) to the corresponding problem $y^{\prime \prime}-y=0, y \in \mathscr{B}$ whose (unique) solution is $y \equiv 0$.

LeMmA 3.4. (i) Suppose $f(t, y, p)$ is continuous on $[0,1] \times$ $R \times R$ and that $M>0$ is such that $y f(t, y, 0)>0$ for $|y|>M$.

(ii) Suppose that there are constants $A, B>0$ such that

$$
|f(t, y, p)| \leqq A p^{2}+B
$$

for all $(t, y)$ in $[0,1] \times[-M, M]$.

Then there are constants $M_{0}=M, M_{1}, M_{2}$ such that for each $\lambda, 0 \leqq \lambda \leqq 1$, and each solution $y$ to $\left(\mathscr{H}_{\lambda}\right)$,

$$
|y(t)| \leqq M_{0}, \quad\left|y^{\prime}(t)\right| \leqq M_{1}, \quad\left|y^{\prime \prime}(t)\right| \leqq M_{2},
$$

for $t$ in $[0,1]$.

Proof. The bounds for $y$ and $y^{\prime}$ are established using (2.1) and (3.1). The bound on $y^{\prime \prime}$ follows from the continuity of $f$ on $[0,1] \times\left[-M_{0}, M_{0}\right] \times\left[-M, M_{1}\right]$. The proof is complete.

4. Existence. Let $\left(C,|\cdot|_{0}\right)$ be the Banach space of continuous functions on $[0,1]$ with the sup norm, $|\cdot|_{0}$. By $\left(C^{n},|\cdot|_{n}\right)$ we denote the Banach space of $n$-times continuously differentiable functions $u \in C$ with the norm,

$$
|u|_{n}=\max \left\{|u|_{0}, \cdots,\left|u^{(n)}\right|_{0}\right\} .
$$

Denote by $\mathscr{B}$ any one of the boundary conditions (I), (II), or (III) and let $C_{0}^{2}=\left\{u \in C^{2}: u \in \mathscr{B}\right\}$. Consider the linear operator $L: C_{0}^{2} \rightarrow C$ given by $u \rightarrow u^{\prime \prime}-u$, the family of maps

$$
T_{\lambda}: C^{1} \longrightarrow C, \quad 0 \leqq \lambda \leqq 1,
$$

defined by

$$
\left(T_{\lambda} v\right)(t)=\lambda\left[f\left(t, v(t), v^{\prime}(t)\right)-v(t)\right],
$$

and the completely continuous embedding $j: C_{0}^{2} \rightarrow C^{1}$.

Let 


$$
r=1+\max \left\{M_{0}, M_{1}, M_{2}\right\}
$$

where $M_{0}, M_{1}, M_{2}$ are the constants of (3.4), and let

$$
K_{r}=\left\{u \in C_{0}^{2}:|u|_{2} \leqq r\right\} .
$$

Assume below that $f(t, y, p)$ satisfies (i) and (ii) of (3.4).

The existence of solutions to problems (I), (II), and (III) will be established by means of a topological transversality theorem. For the definitions of compact homotopy, essential map, and for the full statements of the topological results used in this section, see [3].

Since the operator $L: C_{0}^{2} \rightarrow C$ is invertible, we can define a homotopy $H_{\lambda}: K_{r} \rightarrow C_{0}^{2}$ by,

$$
H_{\lambda}=L^{-1} T_{\lambda} j .
$$

It is easily seen that the fixed points of $H_{\lambda}$ are precisely the solutions of the problem $\left(\mathscr{C}_{\lambda}\right)$; therefore, by the choice of $r$ and (3.4) the homotopy $H_{\lambda}$ is fixed point free on the boundary of $K_{r}$. Moreover, (because of the complete continuity of $j$ ) the homotopy $H_{\lambda}$ is compact. Hence, $H_{0}$ is homotopic to $H_{1}, H_{0} \sim H_{1}$. Since $H_{0}$ is a constant map ( $H_{0}$ is the zero map) it is essential, and, because $H_{1} \sim H_{0}, H_{1}$ is also essential (cf. [3], Theorem 3). In particular $H_{1}$ has a fixed point. This fixed point is a solution to $\left(\mathscr{H}_{1}\right)$, i.e., a solution to problem (I), (II), or (III) depending on the choice of $\mathscr{B}$. Thus, we have proven:

THEOREM 4.1. (i) Suppose that $f(t, y, p)$ is continuous on $[0,1] \times R \times R$ and that there is a constant $M>0$ such that $y f(t, y, 0)>0$ for $|y|>M$.

(ii) Suppose that,

$$
|f(t, y, p)| \leqq A(t, y) p^{2}+B(t, y),
$$

where $A(t, y), B(t, y)>0$ are functions bounded for $(t, y)$ in $[0,1] \times$ $[-M, M]$.

Then each of problems (I), (II), and (III) has at least one solution $y$ in $C^{2}[0,1]$.

5. Uniqueness. We discuss uniqueness theorems for problems (I), (II), and (III) under certain differentiability assumptions, and also under a monotonicity assumption on $f$.

First assume $f(t, y, p)$ is continuous, has bounded first partial derivatives with respect to $y$ and $p$, and satisfies $f_{y} \geqq 0$ for $(t, y, p)$ in $[0,1] \times R \times R$. 
Let $y_{1}$ and $y_{2}$ both be solutions to problem (I) or (II) or (III). Then their difference $y=y_{1}-y_{2}$ satisfies the same boundary conditions as $y_{1}, y_{2}$ as well as the linear differential equation

$$
y^{\prime \prime}=\bar{f}_{y} y+\bar{f}_{p} y^{\prime},
$$

where the bar indicates that $f_{y}$ and $f_{p}$ are evaluated at intermediate points (depending on $y_{1}$ and $y_{2}$ ).

The maximum principle (see [4]) implies:

Lemma 5.1. The difference $y=y_{1}-y_{2}$ cannot achieve a positive (local) maximum or a negative (local) minimum in $(0,1)$ unless it is identically constant.

THEOREM 5.2. Suppose $f(t, u, p)$ is continuous, has bounded first partial derivatives with respect to $y$ and $p$, and satisfies $f_{y} \geqq 0$ for $(t, y, p)$ in $[0,1] \times R \times R$. Then problem (I) has at most one solution.

Proof. The Dirichlet boundary conditions imply that $y$ assumes its extreme values in the interior of $(0,1)$. If $y \neq 0$, then $(5.1)$ implies that $y$ is constant. This constant must be zero, a contradiction. Thus, $y \equiv 0$ and uniqueness is proven.

Uniqueness need not hold for the Neumann and periodic boundary value problems. We can prove:

THEOREM 5.3. Suppose $f$ satisfies the conditions of (5.2). Then any two solutions to problem (II) or problem (III) differ by a constant. If, in addition, $f_{y}\left(t_{0}, y, p\right)>0$ for a fixed $t_{0}$ in $[0,1]$, then problems (II) and (III) have at most one solution.

The proof of (5.3) is based on the following lemma, a result of independent interest which is also used to obtain uniqueness theorems for special cases of problems (IV)-(VII) formulated in $\S 6$.

LEMMA 5.4. If the difference $y=y_{1}-y_{2}$ satisfies either the boundary conditions $y(0)=0, y^{\prime}(1)=0$ or the conditions $y^{\prime}(0)=0$, $y(1)=0$, then $y \equiv 0$.

It is evidently sufficient to consider the boundary conditions $y(0)=0, y^{\prime}(1)=0$. The other case may be treated similarly. If $y(1)=0, y \equiv 0$ by the proof of (5.2). Assume $y(1) \neq 0$. Without loss of generality $y(1)>0$. (If not replace $y$ by $-y$.) By (5.1) 
either $y(1)$ is the (positive) maximum value of $y$ or $y$ is constant. In the latter case $y \equiv y(0)=0$ and the proof is complete. Thus, we assume the former case to hold.

Furthermore, $y$ cannot assume negative values by (5.1) because $y(0)=0<y(1)$. Thus, $y \geqq 0$ in $[0,1]$. Since $y(0)<y(1)$ there is a point $t_{0}$ in $(0,1)$ with $y^{\prime}\left(t_{0}\right)>0$. By applying the maximum principle to a subinterval of $\left[t_{0}, 1\right]$ it is easily seen that $y^{\prime} \geqq 0$ on $\left[t_{0}, 1\right]$. By assumption there is a bound $B$ such that $\left|\vec{f}_{p}\right| \leqq B$ on $[0,1]$. Since $y \geqq 0$ and $y^{\prime} \geqq 0$ on $\left[t_{0}, 1\right]$, we have on $\left[t_{0}, 1\right]$,

$$
y^{\prime \prime}=\bar{f}_{y} y+\bar{f}_{p} y^{\prime} \geqq-B y^{\prime},
$$

and since $y^{\prime}(1)=0$,

$$
\begin{gathered}
y^{\prime}(t) \leqq B \int_{t}^{1} y^{\prime}(x) d x, \\
\left(-e^{B t} \int_{t}^{1} y^{\prime}(x) d x\right)^{\prime} \leqq 0 .
\end{gathered}
$$

Another integration from $t$ to 1 yields

$$
e^{B t} \int_{t}^{1} y^{\prime}(x) d x \leqq 0
$$

Since $y^{\prime} \geqq 0$ on $\left[t_{0}, 1\right]$, this implies $y^{\prime} \equiv 0$ on $\left[t_{0}, 1\right]$. Consequently, $y\left(t_{0}\right)=y(1)$ the positive maximum of $y$ on $[0,1]$. Then (5.1) implies that $y$ is constant. Thus, $y \equiv y(0)=0$, and the lemma is proven.

Proof of 5.3. Let $y=y_{1}-y_{2}$ be the difference of two solutions to the Neumann problem (II). If $y(0)=0$ or $y(1)=0$ then by (5.4) $y \equiv 0$. Thus, we may assume $y(0), y(1) \neq 0$ and without loss of generality that $y(0)>0$. If $y\left(t_{0}\right)=0$ for some $t_{0}$ in $(0,1)$, then $y \equiv 0$ by (5.4) applied to the interval $\left[0, t_{0}\right]$. This contradicts $y(0) \neq 0$; hence, no such $t_{0}$ exists and $y>0$ on $[0,1]$. Assume $y(0)>y(1)$. Then there is a point $t_{0}$ in $(0,1)$ with $y^{\prime}\left(t_{0}\right)<0$. We must have $y^{\prime} \leqq 0$ on $\left[0, t_{0}\right]$; otherwise the maximum principle in contradicted on the subinterval of $\left[0, t_{0}\right]$. Starting with the inequality $y^{\prime \prime} \geqq B y^{\prime}$ which holds on $\left[0, t_{0}\right]$ because $\bar{f}_{y} y \geqq 0$ and arguing along the lines used in the proof of (5.4) we obtain

$$
e^{-B t} \int_{0}^{t} y^{\prime}(x) d x \geqq 0
$$

for $t \in\left[0, t_{0}\right]$. Since $y^{\prime} \leqq 0$ on $\left[0, t_{0}\right]$, this implies $y^{\prime} \equiv 0$ on $\left[0, t_{0}\right]$. Then $y\left(t_{0}\right)=y(0)$ and $y(0)$ must be the positive maximum of $y$ because $y(0)>y(1)$. This contradicts (5.1). So $y(0)>y(1)$ is impossible and, likewise, $y(0)<y(1)$ cannot hold. Thus, $y(0)=y(1)$. Either 
$y \equiv y(0)$ or $y$ assumes a positive minimum value strictly less than $y(0)$ at some point $t_{0}$ in $(0,1)$. In the latter case, $y\left(t_{0}\right)<y(0)$ and $y^{\prime}\left(t_{0}\right)=0=y^{\prime}(0)$. This is impossible by the argument just used when applied to the interval $\left[0, t_{0}\right]$. Hence, $y \equiv y(0)$, a constant, and the first part of (5.3) is established for problem II.

Next assume $y=y_{1}-y_{2}$ where $y_{1}$ and $y_{2}$ satisfy the periodic boundary conditions (III). If $y^{\prime}(0) \neq 0$, then $y$ must assume either a positive maximum of a negative minimum in $(0,1)$. Then by $(5.1)$ $y$ is constant. On the other hand, if $y^{\prime}(0)=0, y$ satisfies problem (II), and $y$ is constant by what has just been proved. This completes the proof of the first part of (5.3).

If $y=y_{1}-y_{2}$ is formed for either problem (II) or (III), then the differential equation satisfied by $y$ reduces to $0=\bar{f}_{y} y$. The final assertion in Theorem 3 follows at once and the proof is complete.

Next uniqueness is proven under a monotonicity condition.

THEOREM 5.5. Suppose that $f(t, y, p)$ is continuous and strictly increasing in $y$ for each fixed $(t, p)$. Then there is at most one solution to each of problems (I), (II), and (III).

The proof is based on the following lemma, which also plays a similar role in $\S 6$ as (5.4).

LEMMA 5.6. Let $f$ satisfy the hypothesis of Theorem 4 and let $y=y_{1}-y_{2}$ be the difference of any two solutions to $z^{\prime \prime}=f\left(t, z, z^{\prime}\right)$. Then $y$ cannot assume a positive (local) maximum or a negative (local) minimum at a point $t_{0}$ in $(0,1)$. The same assertion holds when $t_{0}=0$ or 1 provided that $y^{\prime}\left(t_{0}\right)=0$.

Proof. Suppose $y$ has a positive maximum at $t_{0}$. Then,

$$
\begin{aligned}
& y\left(t_{0}\right)=y_{1}\left(t_{0}\right)-y_{2}\left(t_{0}\right)>0, \\
& 0 \geqq y^{\prime \prime}\left(t_{0}\right)=f\left(t_{0}, y_{1}\left(t_{0}\right), C\right)-\left(f\left(t_{0}, y_{2}\left(t_{0}\right), C\right)\right)>0
\end{aligned}
$$

where $C=y_{1}^{\prime}\left(t_{0}\right)=y_{2}^{\prime}\left(t_{0}\right)$. (The inequalities are reversed at a negative minimum.) This contradiction proves the lemma.

Proof of 5.4. Suppose $y_{1}, y_{2}$ both satisfy problem (I) or (III), and let $y=y_{1}-y_{2}$. Then the boundary conditions imply that $y$ has either a positive maximum or a negative minimum in $(0,1)$ unless $y \equiv 0$. Thus, $y \equiv 0$ by (5.6).

Next assume that $y_{1}, y_{2}$ satisfy problem (II). If $y \not \equiv 0$, then it achieves either a positive maximum or negative minimum in $[0,1]$. This is incompatible with (5.6) under the Neumann conditions. Thus, $y \equiv 0$ and the lemma is proved. 
6. Other boundary value problems. In this section we indicate how the preceding results can be obtained for other important boundary value problems. Consider the following boundary conditions. Conditions (IV)-(VI) are called elastic or Sturm-Liouville conditions, and (VII) defines antiperiodic boundary conditions:

$$
\left\{\begin{array}{rc}
-\alpha y(0)+\beta y^{\prime}(0)=0, & \alpha, \beta>0, \\
a y(1)+b y^{\prime}(1)=0, & a, b>0 .
\end{array}\right.
$$

(a) $y(0)=0, \quad a y(1)+b y^{\prime}(1)=0$

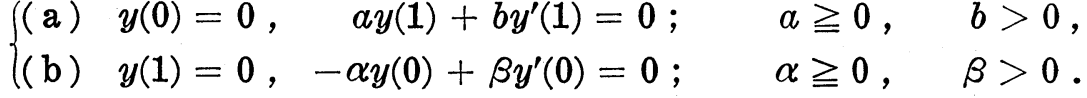

$\left\{\right.$ (a) $y^{\prime}(0)=0, \quad a y(1)+b y^{\prime}(1)=0 ; \quad a>0, \quad b \geqq 0$,

$\left\{\right.$ (b) $y^{\prime}(1)=0, \quad-\alpha y(0)+\beta y^{\prime}(0)=0 ; \quad \alpha>0, \quad \beta \geqq 0$.

(VII) $\left\{\begin{aligned} y(0) & =-y(1), \\ y^{\prime}(0) & =-y^{\prime}(1) .\end{aligned}\right.$

The following results hold:

THEOREM 6.1. If the hypotheses (i) and (ii) of (4.1) hold, then each of problems (IV), (V), (VI) and (VII) has at least one solution $y$ in $C^{2}[0,1]$.

THEOREM 6.2. If the hypothesis of (5.2) or of (5.4) holds, then each of problems (IV), (V), (VI), and (VII) has at most one solution.

REMARK. The uniqueness assertion holds in each case for the class of functions within which it is natural to seek a solution (cf., the Remark in $\S 1$.

The proofs for (6.1) and (6.2) follow closely those for (4.1), (5.2), and (5.5). Thus, we only sketch the arguments.

(a) A priori bounds. For problems (IV) and (V) with $a, \alpha>0$ the boundary conditions force nonzero extreme values to be assumed in $(0,1)$. Thus, a priori bounds on $y$ follow from (2.1). For problems (V) with $a=0, \alpha=0$, (VI), and (VII), the assertion (i) in the proof of (2.2) holds by essentially the same argument. Then (i) and (2.1) yield a priori bounds on $y$ for these problems.

Each of the boundary conditions (IV)-(VII) imply that the derivative of any solution $y$ to the corresponding boundary value problem must vanish at least once in $[0,1]$. A priori bounds on these derivatives follow from (3.1).

The a priori bounds in (3.4) now hold for the family $\left(\mathscr{H}_{\lambda}\right)$, where the boundary conditions $\mathscr{B}$ refer to (IV)-(VII).

(b) Existence. No change is needed in the existence proof. 
(c) Uniqueness. For problem (IV) and (V) when $\alpha, \alpha>0$, the argument used to prove (5.2) can be used. When $a=0, \alpha=0$ in problem (V), (5.4) applies. In problem (VI, a) the type of argument used to prove (5.3) shows that $y=y_{1}-y_{2}$ is constant. The boundary condition at $t=1$ then gives $y \equiv y(1)=0$. Uniqueness follows similarly for (VI, b). Finally, for the antiperiodic problem (VII), $y=y_{1}-y_{2}$ must be constant just as for the periodic case; however, this constant must be zero because $y(0)=-y(1)$.

To establish uniqueness under the monotonicity condition in (5.5) simply use (5.6).

7. Examples. In this section several examples are given to illustrate the preceding results.

(a) Boundary value problems of the type considered above occur frequently in mathematical physics. For instance, a steady state temperature distribution, $y$, in a rod (identified with the closed unit interval) is governed by the differential equation,

$$
\left(k y^{\prime}\right)^{\prime}+q(t, y)=0
$$

where $k=k(t, y)>0$ is the thermal conductivity at position $t$ and temperature $y$, and $q(t, y)$ describes internal heat sources. The differential equation may be expressed as $y^{\prime \prime}=f\left(t, y, y^{\prime}\right)$ where

$$
f\left(t, y, y^{\prime}\right)=\frac{-1}{k(t, y)}\left[k_{t} y^{\prime}+k_{y} y^{\prime 2}+q(t, y)\right]
$$

and the subscripts denote partial derivatives. Assume that $k$ is a continuously differentiable function of its arguments and that $q$ is continuous.

If $y q(t, y)<0$ for $|y|$ sufficiently large, then by (4.1) and (6.1) a steady state solution exists for any choice of boundary conditions (I)-(VI). A common choice for $q(t, y)$ is,

$$
q(t, y)=r(t) y+s(t)
$$

and which case

$$
y q(t, y)=r(t) y^{2}+s(t) y
$$

Clearly, $y q(t, y)<0$ for large $|y|$ if $r(t)<0$. Physically this corresponds to a governing mechanism in the rod which prevents arbitrarily large temperature extremes. Thus, a steady state solution is expected on physical grounds. If $k$ is constant, then

$$
f_{y}(t, y, p)=-q_{y} / k=-r(t) / k>0
$$

assuming, as above, that $r(t)<0$. In this case (5.3) and (6.2) show 
that the steady state solution is unique for each choice of boundary conditions (I)-(VI).

(b) The Dirichlet problem

$$
\left\{\begin{array}{l}
y^{\prime \prime}=y^{3}+y^{\prime 2}+1 \\
y(0)=0, \quad y(1)=0
\end{array}\right.
$$

has a unique solution by (4.1) and (5.2). (The assumption (i) in (4.1) holds by (2.3).) Note that $f_{y}=3 y^{2}$ here so Bernstein's theorem does not apply.

(c) Let $\mathscr{B}$ denote one of the boundary conditions (I)-(VII). The problem

$$
\left\{\begin{array}{l}
y^{\prime \prime}=\sum_{k=0}^{n} a_{k}\left(t, y^{\prime}\right) y^{k}+b\left(t, y^{\prime}\right) \\
y \in \mathscr{B}
\end{array}\right.
$$

has a solution provided $n$ is odd, the $a_{k}$ and $b$ are continuous,

$$
\begin{aligned}
\left|a_{k}(t, p)\right| & \leqq b_{k} p^{2}+c_{k}, \quad k=0, \cdots, n, \\
|b(t, p)| & \leqq d p^{2}+e,
\end{aligned}
$$

where $b_{k}, c_{k}, d, e$ are constants, and

$$
a_{n}(t, 0)>0, \quad t \text { in }[0,1] .
$$

This follows from (4.1) and (6.1).

(d) The boundary value problem with inhomogeneous boundary conditions,

$$
\left\{\begin{array}{l}
y^{\prime \prime}=f\left(t, y, y^{\prime}\right), \\
-\alpha y(0)+\beta y^{\prime}(0)=C, \quad \alpha y(1)+b y^{\prime}(1)=D
\end{array}\right.
$$

where $\alpha, \beta, a, b \geqq 0$ and $\alpha^{2}>0, a^{2}>0$ has a solution if:

(i )' There is a constant $M \geqq 0$ such that $y f(t, y, 0)>0$ for $|y|>M$.

(ii)' There are functions $A(t, y), B(t, y)>0$ such that

$$
|f(t, y, p)| \leqq A(t, y) p^{2}+B(t, y)
$$

where $A(t, y), B(t, y)$ are bounded on bounded $(t, y)$ sets. This follows as for (4.1) and (6.1) by means of the substitution $u=y-l$ where $l(t)=A t+B$ with $A$ and $B$ defined by

$$
A=\frac{a C+\alpha D}{a \alpha+a \beta+\alpha b} \quad B=\frac{\beta D-(a+b) C}{a \alpha+a \beta+\alpha \beta} .
$$

The linear function $l$ satisfies the inhomogeneous boundary conditions 
and so the original boundary value problem is essentially equivalent to the problem,

$$
\left\{\begin{array}{l}
u^{\prime \prime}=F\left(t, u, u^{\prime}\right) \\
-\alpha u(0)+\beta u^{\prime}(0)=0, \quad a u(1)+b u^{\prime}(1)=0
\end{array}\right.
$$

where $F\left(t, u, u^{\prime}\right)=f\left(t, u+l, u^{\prime}+l^{\prime}\right)$. It is easily verified that (i)' implies $|u|_{0} \leqq M_{0}=\max \{|C / \alpha|,|D / \alpha|, M\}$ for any solution $u$ to this problem. Then, (ii)' implies (ii) of (4.1) for $F$. Thus, the problem for $u$, and hence for $y$, has a solution by the argument of $\S 4$. If $f_{y} \geqq 0$ the solution is unique.

Note that the inhomogeneous boundary conditions covered by this example correspond to the homogeneous boundary conditions (I), (IV), (V) with $\alpha$ and $\alpha>0$, and (VI).

(e) The condition $y f>0$ holds for $|y|>0$ in the following example; however, a priori bounds are not available because $y$ achieves its extrema at 0 and 1 (cf., (2.1)). The problem,

$$
\left\{\begin{array}{l}
y^{\prime \prime}=y \\
y(0)=y(1), \quad y^{\prime}(0)=-y^{\prime}(1)
\end{array}\right.
$$

has solutions

$$
y(t)=A\left(e^{t}+e e^{-t}\right)
$$

where $A$ is any constant.

(f) (4.1) does not guarantee the existence of a solution for,

$$
\left\{\begin{array}{l}
y^{\prime \prime}=y^{\prime 2}+1, \\
y(0)=0, \quad y(1)=0,
\end{array}\right.
$$

however, this problem does have a solution, namely,

$$
y(t)=\log (\cos 1 / 2)-\log (\cos (t-1 / 2)) .
$$

If the differential equation is changed to $y^{\prime \prime}=y^{\prime 2}+\pi^{2}$ then the Dirichlet problem has no solution.

( $g$ ) It is easily checked that the Neumann problem

$$
\left\{\begin{array}{l}
y^{\prime \prime}=y^{\prime 2}+1 \\
y^{\prime}(0)=0, \quad y^{\prime}(1)=0
\end{array}\right.
$$

does not have a solution.

(h) Consider,

$$
\left\{\begin{array}{l}
y^{\prime \prime}=3 y^{\prime}-\sqrt{|y|}+\sin t \\
y(0)=0, \quad y(1)=0
\end{array}\right.
$$


The results above do not apply directly; however, the change of variables $y=e^{t} u$ yields the essentially equivalent problem,

$$
\left\{\begin{array}{l}
u^{\prime \prime}=u^{\prime}+2 u-e^{-(1 / 2) t} \sqrt{|u|}+e^{-t} \sin t, \\
u(0)=0, \quad u(1)=0
\end{array}\right.
$$

which is easily seen to have a solution by (4.1).

(i) Consider

$$
\left\{\begin{array}{l}
a(t) y^{\prime \prime}+b(t) y^{\prime}+c(t) y=f\left(t, y, y^{\prime}\right) \\
y \in \mathscr{B}
\end{array}\right.
$$

where $a(t)>0, b(t)$ and $c(t)$ are continuous on [0,1]. Assume:

(i) There is a constant $M>0$ such that

$$
y[f(t, y, 0)-c(t) y]>0
$$

for $|y|>M$.

(ii) There are functions $A(t, y), B(t, y)>0$ and bounded on $[0,1] \times[-M, M]$ such that

$$
|f(t, y, p)| \leqq A(t, y) p^{2}+B(t, y) .
$$

Then (1) has a solution $y \in C^{2}[0,1]$ for each set $\mathscr{B}$ of boundary conditions (I)-(VII).

This assertion is proved by making minor adjustments to the proofs in $\S \S 2-4$.

\section{REFERENCES}

1. P. B. Bailey, L. F. Shampine, P. E. Waltman, Nonlinear Two Point Boundary Value Problems, Academic Press, New York and London, 1968.

2. S. N. Bernstein, Sur less équations du calcul des variations, Ann. Sci. École Norm. Sup., 29 (1912), 431-485.

3. A. Granas, Sur la méthode de continuité de Poincaré, Comptes Rendus Acad. Sci. Paris, 282 (1976), 983-985.

4. M. H. Protter, H. F. Weinberger, Maximum Principles in Differential Equations, Prentice-Hall, Inc., Englewood Cliffs, New Jersey, 1976.

Received June 3, 1977.

OREgon State University

CoRvallis, OR 97331 


\section{PACIFIC JOURNAL OF MATHEMATICS}

\section{EDITORS}

RICHARD ARENS (Managing Editor)

University of California

Los Angeles, California 90024

C. W. Curtis

University of Oregon

Eugene, OR 97403

C. C. MOORE

University of California

Berkeley, CA 94720

\section{J. DUGUNDJI}

Department of Mathematics University of Southern California Los Angeles, California 90007

R. Finn AND J. Milgram Stanford University Stanford, California 94305

ASSOCIATE EDITORS
E. F. BECKENBACH

B. H. NeUMANN

F. WOLF

K. YoshidA

\section{SUPPORTING INSTITUTIONS}

UNIVERSITY OF BRITISH COLUMBIA UNIVERSITY OF SOUTHERN CALIFORNIA CALIFORNIA INSTITUTE OF TECHNOLOGY STANFORD UNIVERSITY UNIVERSITY OF CALIFORNIA MONTANA STATE UNIVERSITY UNIVERSITY OF TOKYO UNIVERSITY OF NEVADA, RENO UNIVERSITY OF UTAH NEW MEXICO STATE UNIVERSITY OREGON STATE UNIVERSITY UNIVERSITY OF OREGON

WASHINGTON STATE UNIVERSITY UNIVERSITY OF WASHINGTON OSAKA UNIVERSITY 


\section{Pacific Journal of Mathematics}

\section{Vol. 74, No. $1 \quad$ May, 1978}

Gerald Arthur Anderson, Computation of the surgery obstruction groups

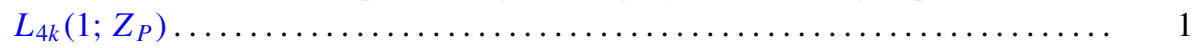

R. K. Beatson, The degree of monotone approximation ................ 5

Sterling K. Berberian, The character space of the algebra of regulated functions . . . 15

Douglas Michael Campbell and Jack Wayne Lamoreaux, Continua in the plane with

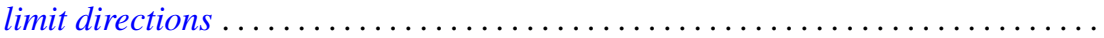

R. J. Duffin, Algorithms for localizing roots of a polynomial and the Pisot

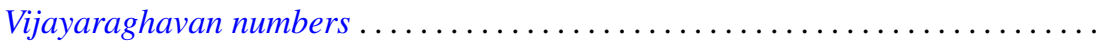

Alessandro Figà-Talamanca and Massimo A. Picardello, Functions that operate on

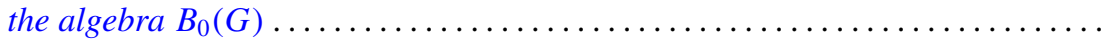

John Erik Fornaess, Biholomorphic mappings between weakly pseudoconvex

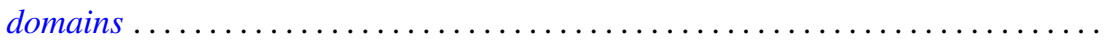

Andrzej Granas, Ronald Bernard Guenther and John Walter Lee, On a theorem of S.

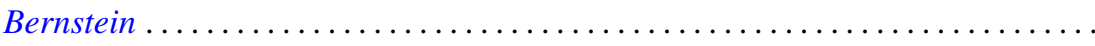

Jerry Grossman, On groups with specified lower central series quotients . .........

William H. Julian, Ray Mines, III and Fred Richman, Algebraic numbers, a constructive development . . . . . . . . . . . . . . . . . . . . . . .

Surjit Singh Khurana, A note on Radon-Nikodým theorem for finitely additive

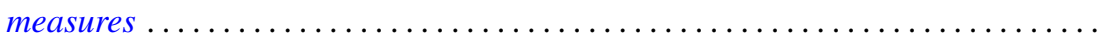

Garo K. Kiremidjian, A Nash-Moser-type implicit function theorem and nonlinear

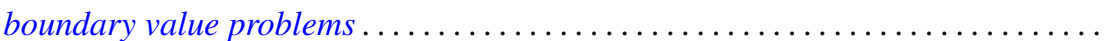

Ronald Jacob Leach, Coefficient estimates for certain multivalent functions ....

John Alan MacBain, Local and global bifurcation from normal eigenvalues. II . . 133

James A. MacDougall and Lowell G. Sweet, Three dimensional homogeneous algebras...

John Rowlay Martin, Fixed point sets of Peano continua ......

R. Daniel Mauldin, The boundedness of the Cantor-Bendixson order of some analytic sets...

Richard C. Metzler, Uniqueness of extensions of positive linear functions ..

Rodney V. Nillsen, Moment sequences obtained from restricted powers . .

Keiji Nishioka, Transcendental constants over the coefficient fields in differential elliptic function fields...

Gabriel Michael Miller Obi, An algebraic closed graph theorem

Richard Cranston Randell, Quotients of complete intersections by $\mathbf{C}^{*}$ actions . . 221

Bruce Reznick, Banach spaces which satisfy linear identities . .

Bennett Setzer, Elliptic curves over complex quadratic fields...

Arne Stray, A scheme for approximating bounded analytic functions on certain subsets of the unit disc.

Nicholas Th. Varopoulos, A remark on functions of bounded mean oscillation and bounded harmonic functions. Addendum to: "BMO functions and the

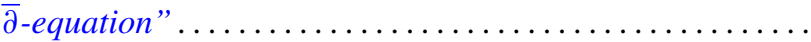

Charles Irvin Vinsonhaler, Torsion free abelian groups quasi-projective over their

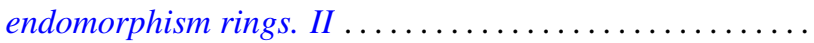

Thomas R. Wolf, Characters of $p^{\prime}$-degree in solvable groups ... 